\title{
The RPC31 Gene of Saccharomyces cerevisiae Encodes a Subunit of RNA Polymerase C (III) with an Acidic Tail
}

\author{
CHRISTINE MOSRIN, ${ }^{1}$ MICHEL RIVA,${ }^{1,2}$ MONICA BELTRAME, ${ }^{3}$ EVELYNE CASSAR, ${ }^{1}$ \\ ANDRÉ SENTENAC, ${ }^{1,2}$ AND PIERRE THURIAUX ${ }^{1.2 *}$ \\ Service de Biochimie ${ }^{1 *}$ and Laboratoire d'Ingénièrie des Protéines, ${ }^{2}$ Commissariat à l'Energie Atomique, \\ Centre d'Etudes Nucléaires de Saclay, F91191 Gif sur Yvette, France, and European Molecular \\ Biology Laboratory, D-6900 Heidelberg, Federal Republic of Germany ${ }^{3}$
}

Received 20 March 1990/Accepted 24 June 1990

\begin{abstract}
The RPC31 gene encoding the C31 subunit of Saccharomyces cerevisiae RNA polymerase C (III) has been isolated, starting from a $\mathrm{C}$-terminal fragment cloned on a $\lambda$ gt11 library. It is unique on the yeast genome and lies on the left arm of chromosome XIV, very close to a NotI site. Its coding sequence perfectly matches the amino acid sequence of two oligopeptides prepared from purified C31. It is also identical to the $A C P 2$ gene previously described as encoding an HMG1-like protein (W. Haggren and D. Kolodrubetz, Mol. Cell. Biol. 8:1282-1289, 1988). Thus, $A C P 2$ and $R P C 31$ are allelic and encode a subunit of RNA polymerase C. The c31 protein has a highly acidic C-terminal tail also found in several other chromatin-interacting proteins, including animal HMG1. Outside this domain, however, there is no appreciable homology to any known protein. The growth phenotypes of a gene deletion, of insertions, and of nonsense mutations indicate that the C31 protein is strictly required for cell growth and that most of the acidic domain is essential for its function. Random mutagenesis failed to yield temperature-sensitive mutants, but a slowly growing mutant was constructed by partial suppression of a UAA nonsense allele of $R P C 31$. Its reduced rate of tRNA synthesis in vivo relative to 5.8S rRNA supports the hypothesis that the C31 protein is a functional subunit of RNA polymerase $\mathrm{C}$.
\end{abstract}

The transcription of the eucaryotic genome is catalyzed by three distinct RNA polymerases, $\mathrm{A}, \mathrm{B}$, and $\mathrm{C}$, acting in conjunction with their transcription factors. Enzyme A (or I) synthesizes the large nucleolar precursor of rRNA, enzyme B (or II) synthesizes the mRNAs, and enzyme C (or III) synthesizes 5S rRNA, tRNAs, and a few other small, untranslated RNA species. Unlike the bacterial enzyme, eucaryotic RNA polymerases have no template specificity but select the appropriate class of promoters by recognizing specific preinitiation complexes formed between the promoter and cognate transcription factors (TFIIIA and TFIIIC in the case of enzyme $C$ ). They have a much more complex subunit structure than does the bacterial RNA polymerase, since the yeast enzymes dissociate into 12 distinct polypeptidic components under denaturing conditions. This astonishing structural complexity is still poorly understood in functional terms $(11,43,44)$.

Biochemical and genetic studies of yeast polymerases have suggested a minimal subunit structure (38) formed of the two large subunits strongly related to the bacterial $\beta^{\prime}$ and $\beta$ subunits $(1,28,46)$, three small subunits (ABC27, ABC23, and $\mathrm{ABC} 14)$ common to all three enzymes (18), and at least one subunit (AC40) (25) identical in enzymes $A$ and $C$ and structurally equivalent to the B44 subunit of enzyme B (23). Another subunit shared by enzymes A and C (AC19) probably has no counterpart in enzyme $B$ (18). In addition, each RNA polymerase contains several specific subunits. There are at least four of them $(\mathrm{C} 80, \mathrm{C} 53, \mathrm{C} 34$, and C31) in enzyme $\mathrm{C}$ (18) and probably also four in RNA polymerase A (38). In the latter case, the specific subunits are polymorphic even between closely related yeast species such as Saccharomyces cerevisiae and $S$. douglasii (38). The functional role of these small and specific subunits in the cognate RNA poly-

\footnotetext{
* Corresponding author.
}

merase remains to be understood and was genetically approached in the present work by identifying and characterizing the gene encoding the $C 31$ subunit of yeast RNA polymerase $\mathrm{C}$.

\section{MATERIALS AND METHODS}

Strains and media. Strains are listed in Table 1. Yeast genetic techniques and media were described by Sherman et al. (45). Except for tryptophan, the amino acid requirements were also met by adding $0.1 \%$ Casamino Acids to the standard minimal medium. Reduction in the wild-type growth rate was obtained by adding $2 \%$ sterile filtered glucosamine to the Casamino Acids medium containing $0.5 \%$ glucose as the carbon source (27). The 5-fluoro-orotic acid medium was described by Boeke et al. (4). For experiments with Escherichia coli, strain DH5 $\mathrm{FF}^{\prime} \mathrm{I}^{\mathrm{q}}$, obtained from Bethesda Research Laboratories, Inc., was used. Bacterial media were as described by Davis et al. (8).

Plasmid constructions. The RPC31 plasmids pJC31, pC326, pC329, and pSE359-RPC31 were constructed by inserting the 2.5-kilobase (kb) EcoRI fragment spanning RPC31 (isolated from plasmid YCp50-C31; see Fig. 1) into the pJDB110 (33), pEMBLYCp32, pFL39, and pSE359 vectors. Plasmid pEMBLYCp32 (constructed by $C$. Mann) is pEMBLYi32 $(2,14)$ with a 1.4-kb ARSI-CENIV cassette inserted at the AatII site. Plasmid pFL39 is a centromeric vector obtained from $F$. Lacroute. It harbors a $0.83-\mathrm{kb}$ EcoRI-PstI TRPI fragment (without $A R S I$ ) and a $0.8-\mathrm{kb}$ TaqI fragment containing $C E N V I$ and an uncharacterized $A R S$ fragment. Plasmid pSE359 (obtained from C. Mann) is a 5.3-kb YRp vector derived from TRPI YRp7 (33). It has an EcoRI, SacI, BamHI, SalI, and SphI multiple cloning site and bears the SUPM11-o ochre (UAA) supressor allele corresponding to a tRNA $^{\text {Tyr }}$ mutated at the anticodon. Plasmid pC50 was constructed by inserting the 2.1-kb SpeIEcoRI RPC31 fragment into the centromeric vector pUN50 
TABLE 1. Yeast strains

\begin{tabular}{|c|c|c|}
\hline Strains & Genotype $^{a}$ & Source or reference ${ }^{b}$ \\
\hline YNN281 & MATa ade2-101 ura3-52 lys2-801 his $3 \Delta 200$ trpl- $\Delta$ & YGSC \\
\hline YNN282 & MAT $\alpha$ ade2-101 ura3-52 lys2-801 his $3 \Delta 200$ trpl- $\Delta$ & YGSC \\
\hline D27-7c & MATa ade2-101 ura3-52 lys2-801 his $3 \Delta 200$ trp1-901 leu2-3,112 rpc160-41 pep4::HIS3 & 14 \\
\hline D34-3a & MAT $\alpha$ ade2-101 ura3-52 lys2-801 his $3 *$ trpl- $\Delta 800$ leu2-3,112 & This work \\
\hline D34-3b & MATa ade2-101 ura3-52 lys2-801 his3* trpl $\Delta 800$ leu2-3,112 & This work \\
\hline CDM62 & 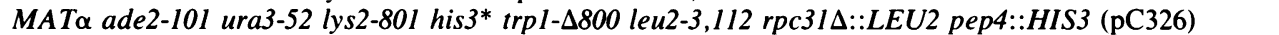 & This work \\
\hline DM824 & MAT $\alpha$ ade2-101 ura3-52 lys2-801 his3* trpl 4800 leu2-3,112 rpc31D::LEU2 pep4::HIS3 (pSE359-Tyr4) & This work \\
\hline Le788 & MAT $\alpha$ ura 3 met 2 met4 lys 10 arg8 adel his6 pha2 & F. Hilger \\
\hline $\mathrm{KJ} 382-23 \mathrm{~A}$ & MATa spoll ura3 cyh2 ade2 his7 home3 tyrl canl RPC3::pJC31 & This work \\
\hline DM32-1 & MATa ura3-52 trpl RPC31::Y1pC327 & This work \\
\hline DM33-1a & MAT $\alpha$ ura3* met 2 pha2 his6 adel & This work \\
\hline DM34-7d & MAT $\alpha$ trpl RPC31::Y1pC327 ura3* & This work \\
\hline D55-7b & MATa ura $^{*}$ met 4 petx ade2 & This work \\
\hline
\end{tabular}

${ }^{a}$ his3* is either his $3 \Delta 200$ or his3-11,15; ura3* is an unidentified ura3 allele (possibly ura3-52).

${ }^{b}$ YGSC, Yeast Genetic Stock Center, Berkeley, Calif.

(9). To construct pBSC31-5, we digested plasmid pC329 with NotI, subjected it to a brief digestion with exonuclease III, and treated it with mung bean nuclease. The plasmid was further cut with EcoRI, and the small 1.2-kb fragment was ligated to the pBluescript $\mathrm{KS}$ (Stratagene) plasmid. The resulting plasmid (pBSC31-5) bearing no ATG before the first ATG of the C31 coding region was used for in vivo transcription and translation experiments as recommended by Stratagene (transcription) and by Promega Biotech (translation).

Disruption of the RPC31 gene. The $r p c 31:: H I S 3 a$ and $r p c 31:: H I S 3 b$ alleles were produced by inserting the HIS3 gene (a 1.7-kb BamHI fragment of plasmid pSZ63 [33]) in both orientations at the BamHI site of RPC31. The last 6 amino acids of the $R P C 31$ coding sequence were replaced by 30 others in rpc31::HIS3a and by 6 unrelated amino acids in $r p c 31:: H I S 3 b$ (see Fig. 3). rpc31 $:: L E U 2$ was constructed by replacing the 810-base-pair (bp) SalI fragment of RPC3I with the 2.2-kb XhoI-SalI LEU2 marker from YEp13 (33). Heterozygous $R P C 31 / r p c 31:: \Delta L E U 2$ or RPC 31/rpc $31:: H I S 3$ diploids were constructed in strain YNN281 $\times$ YNN282 by the allele replacement technique (42). Their genetic structures were confirmed by Southern analysis with the $2.1-\mathrm{kb}$ XbaI-SalI RPC31 fragment (see Fig. 1) as a probe. The $r p c 31:: \operatorname{Tn} 10-13,-33$, and -38 alleles were obtained by transposon mutagenesis (19). Their approximate insertion sites were determined by restriction mapping. Tn10-33 appeared to be inserted about 100 bp upstream from the RPC 31 coding sequence itself. Tn10-13 generated an N-terminal fusion of lacZ to RPC31, as shown by the blue color of the corresponding mutant on YPD supplemented with 5-bromo-4chloro-3-indolyl- $\beta$-D-galactopyranoside (X-Gal) at $40 \mathrm{mg}$ ) liter.

In vitro mutagenesis. Plasmid pC329 (TRPI RPC3I) or pC50 (TRPI RPC31) was mutagenized with hydroxylamine (40) and directly introduced into the $\operatorname{rpc} 31 \Delta:: L E U 2$ strain CDM62(pC326) by transformation. Each transformant may receive several distinct plasmid molecules (which would prevent the detection of recessive phenotypes), but the cellular plasmid content of individual cells should become homogeneous after a few rounds of mitotic segregation. Accordingly, the initial transformants were pooled and respread on selective medium at appropriate dilutions to form individual colonies, which were then replica plated on 5fluoro-orotic acid medium. Defective mutants are unable to grow on 5-FOA medium, which selects for colonies that have lost $\mathrm{pC} 326$. This selection was done at different tem- peratures $\left(16,25,30\right.$, and $\left.37^{\circ} \mathrm{C}\right)$ to identify conditional mutants in addition to fully defective ones. No conditional RPC31 mutant was obtained, but 11 temperature-sensitive TRP1 mutants were isolated, indicating that plasmid-borne conditional mutants were generated and detected under our experimental conditions. A total of 225 fully defective $r p c 31$ mutants were obtained (corresponding to $1.5 \%$ of the colonies). The mutagenesis was probably close to saturation because $166 \mathrm{GC} \rightarrow \mathrm{AT}$ transitions are possible on the RPC3I coding sequence, of which 45 are likely to be silent mutations, as they would correspond to substitutions in the acidic C-terminal domain (see Results). The six nonsense alleles were constructed by oligonucleotide-directed mutagenesis of the pC50 plasmid with a kit from Amersham Corp. Sequencing of the entire $R P C 31$ coding sequence showed the absence of spurious additional mutations in the corresponding clones.

In vivo labeling of RNAs. Exponential cultures $(10 \mathrm{ml})$ on Casamino Acids medium supplemented with $0.02 \%$ uracil and tryptophan were transferred by filtration to a medium containing $5 \times 10^{5} \mathrm{~Bq}$ of tritiated uracil at a final concentration of $0.5 \mathrm{mg} /$ liter. After $15 \mathrm{~min}$ of growth, the culture was chilled and harvested by centrifugation at $4^{\circ} \mathrm{C}$. The pellet was suspended in $2.5 \mathrm{ml}$ of cold TE buffer $(10 \mathrm{mM}$ Tris hydrochloride [pH 7.4], $10 \mathrm{mM}$ EDTA) with $0.5 \%$ sodium dodecyl sulfate, and the nucleic acids were extracted in the same volume of phenol (saturated in TE buffer) under vigorous agitation at $65^{\circ} \mathrm{C}$ for $1 \mathrm{~h}$. The aqueous phase was precipitated with sodium acetate (final concentration, $0.3 \mathrm{M}$ ) plus 2.5 volumes of ethanol. The pellet was ethanol washed, dried, and suspended in $200 \mu$ l of water. The yield was about $200 \mu \mathrm{g}$ of RNA, as measured by UV $A_{260}$ and $A_{280}$. RNAs were separated by electrophoresis on a $6 \%$ polyacrylamide gel as previously described (14).

DNA sequences. Overlapping restriction subfragments of the 2.5-kb EcoRI fragment (see Fig. 1) were inserted in the $\mathrm{pBS}^{+}$or $\mathrm{pBS}^{-}$plasmid (Stratagene). The sequence was determined on both strands by the dideoxy method with modified T7 DNA polymerase (Sequenase, United States Biochemical Corp.). Open reading frame and acid-baseamino acid mapping was done with the DNA Strider program (26). Searches of data bases, secondary structure predictions, and sequence alignments were performed at computer facilities at CITI2, Paris, France, on a VAX8530 computer (Ministère de la Recherche et de la Technologie) with the FASTA package program of Pearson and Lipman (34). 


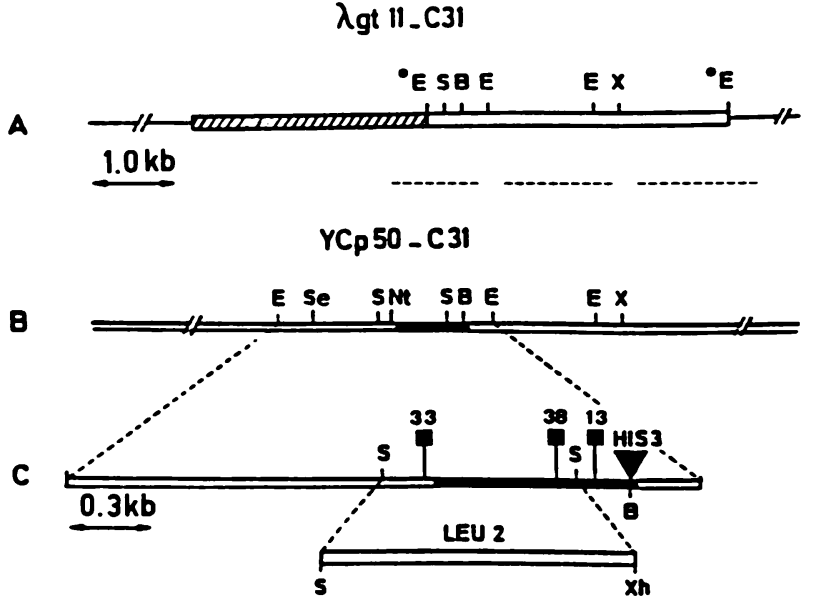

FIG. 1. Restriction map of the RPC31 region. (A) $\lambda$ gt11-C31 insert $(3.6 \mathrm{~kb})$, with approximate locations of three transcripts, as deduced from Fig. 2. (B) YCp50-C31 insert (13.5 kb). (C) Expanded view of the 2.5-kb EcoRI fragment spanning RPC31, with approximate locations of deletion and insertion mutations. 33, 38, and 13 indicate three insertions of the $\operatorname{Tn} 10-L U K\left(U R A 3^{+}\right)$minitransposon. HIS3 indicates the 1.7-kb yeast BamHI HIS3 cassette inserted in either orientation and leading to a recessive-lethal $(r p c 31:: H I S 3 a)$ or viable (rpc31::HIS3b) phenotype (see also Fig. 3). LEU2 indicates a Sall-XhoI $(2.2-\mathrm{kb})$ cassette. Solid bars indicate the RPC3I coding sequence. Open boxes indicate yeast genomic DNA outside the $R P C 31$ gene. The hatched box indicates the lacZ DNA on $\lambda \mathrm{gt} 11$. Thin lines indicate vector DNA. Broken lines indicate approximate locations of the three transcripts hybridized by the $\lambda$ gt11-C31 DNA (see Fig. 2). Restriction sites: B, BamHI; E, EcoRI; Nt, NotI; S, SalI; Se, SpeI; X, XbaI; Xh, XhoI. *E denotes artificial EcoRI sites created by the construction of the $\lambda \mathrm{gt} 11-\mathrm{C} 31$ clone.

Microsequencing of oligopeptides. RNA polymerase $\mathrm{C}$ prepared as described by Huet et al. (18) showed the usual polypeptide pattern except that the 37-kilodalton polypeptide was absent from the preparation. The enzyme $(2 \mathrm{mg})$ was loaded on a $12 \%$ polyacrylamide gel, and the subunits were separated by electrophoresis under denaturing conditions. The band containing the $\mathrm{C} 31$ subunit was cut from the gel and incubated with tolylsulfonyl phenylalanyl chloromethyl ketone-treated trypsin for $18 \mathrm{~h}$ at $37^{\circ} \mathrm{C}$ in $0.1 \mathrm{M}$ ammonium acetate $(\mathrm{pH} \mathrm{8.3)}$ at a protein/enzyme ratio of 1:50 (wt/wt). The peptides were separated by high-pressure liquid chromatography on an Aquapore RP300 reversed-phase column (10 cm by $2.1 \mathrm{~mm}$; Brownlee Laboratories). The column was developed at $40^{\circ} \mathrm{C}$ with a linear gradient of 25 $\mathrm{mM}$ ammonium acetate (pH 6.2) to $60 \%$ acetonitrile- $50 \mathrm{mM}$ ammonium acetate $\left(\mathrm{pH} \mathrm{6.2)}\right.$ at $200 \mu \mathrm{l} / \mathrm{min}$ for $45 \mathrm{~min} . A_{214}$ peaks were collected and chromatographed in a second system $\left(0.115 \%\right.$ trifluoroacetic acid to $60 \% \mathrm{CH}_{3} \mathrm{CN}-0.1 \%$ trifluoroacetic acid). Fractions corresponding to $A_{214}$ peaks were spotted on a precycled, Polybrene-coated, trifluoroacetic acid-activated glass fiber filter for sequencing in a 477 Sequenator (Applied Biosystems).

\section{RESULTS}

Cloning of gene RPC31. Starting from a previously isolated तgt11-C31 clone (39), the yeast insert (a 3.6-kb fragment delimited by two EcoRI sites generated by the cloning procedure) was subcloned, and an internal 2.1-kb SalI-XbaI fragment (Fig. 1A) was used as a probe to screen a yeast genomic library harbored by the centromeric plasmid YCp50

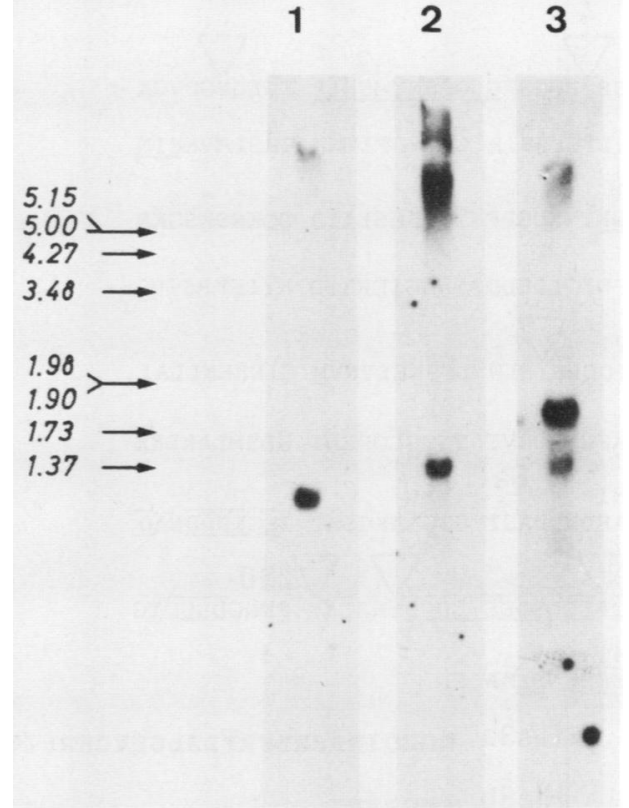

FIG. 2. Northern blot analysis. Yeast RNA $(15 \mu \mathrm{g})$ from strain YNN281 was electrophoresed in a 1\% agarose gel after denaturation with glyoxal, transferred to a nitrocellulose membrane, and hybridized with the three consecutive 0.6-, 1.2-, and 1.8-kb EcoRI fragments (lanes 1, 2, and 3, respectively) of the $\lambda$ gt11-C 31 clone (Fig. 1A) under high-stringency conditions. Denaturated $\lambda$ DNA restriction fragments were used as molecular weight markers. Numbers at left are in kilobases.

(41). One of the plasmids obtained (YCp50-C31) has a 13.5-kb Sau3A insert which contains a NotI site (8 bp) and includes the whole $R P C 31$ gene (Fig. 1B). Northern (RNA) hybridization showed that the initial 3.6-kb insert hybridized to three transcripts of about $1.2,1.5$, and $1.7 \mathrm{~kb}$ (Fig. 2) which could be approximately located as depicted in Fig. 1A. The $1.2-\mathrm{kb}$ transcript corresponded to the $R P C 31$ fragment of $\lambda$ gt11-C 31 . Its size was consistent with a gene product of 31 kilodaltons.

We determined the 1,279-bp sequence of the SalI-EcoRI fragment spanning $R P C 31$ (Fig. 1). The coding sequence (Fig. 3) is identical to that of the $A C P 2$ gene independently isolated by Haggren and Kolodrubetz (15) and interpreted by them as encoding an HMGl-like protein (our arguments against this interpretation are given below, in the amino acid sequence analysis section). The corresponding region is present in a single copy on the yeast genome (Fig. 4), showing that $R P C 31$ and $A C P 2$ are the same gene. To firmly establish that this gene encodes the $\mathrm{C} 31$ protein, we digested a purified preparation of $\mathrm{C} 31$ with trypsin and microsequenced two of the resulting oligopeptides. In both cases, there was a perfect match between the observed and predicted amino acid sequences (Fig. 3). One oligopeptide (GGSNNYMSNDP) included the Met at position 14, allowing us to define the initiator codon as indicated in Fig. 3. The $R P C 31$ reading frame indicates a theoretical $M_{\mathrm{r}}$ of 27,707 , somewhat lower than the $M_{\mathrm{r}}$ of 31,000 indicated by electrophoretic migration in polyacrylamide gels (18). This discrepancy is unlikely to reflect a postranscriptional modification of the gene product, since an apparent $M_{r}$ of 31,000 was also observed when the RPC 31 gene was expressed in vitro from plasmid pBSC32-5 (data not shown). Similar minor discrepancies have been observed for other subunits of RNA polymerase $C(1,25)$. 


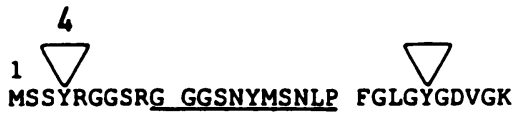

NHITEFPSIP LPINGPITNK ERSLAVKYIN

61 FGKTVKDGPF YTGSMSLIID QQENSKSGKR

KPNIILDEDD TNDGIERYSD KYLKKRKIGI

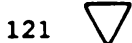

SIDDHPYNLN LFPNELYNVM GINKKKLLAI

SKFNNADDVF TGTGLQDENI GLSMLAKLKE

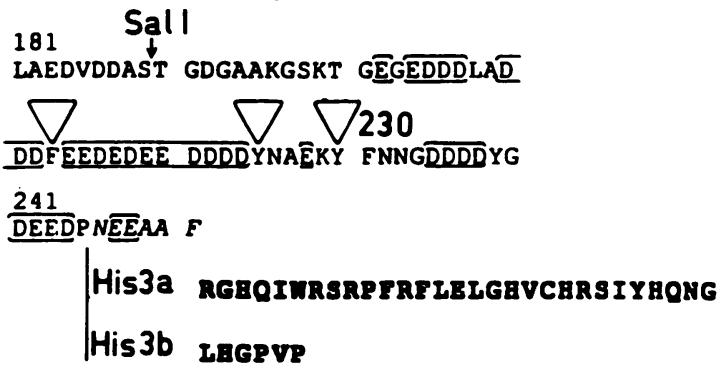

FIG. 3. Amino acid sequence of the RPC3I gene. The two oligopeptides determined by microsequencing are underlined. The Asp-Glu acidic tail is bracketed. The Sall site defining the border of the two DNA probes used in Fig. 4 is indicated by an arrow. The predicted amino acid substitutions generated by the $\operatorname{rpc} 31:: H I S 3 a$ (lethal) and $r p c 31:: H I S 3 b$ (viable) insertions are shown in boldface type (see the text). Open triangles represent substitutions of UAU (Tyr) codons by a nonsense UAA (rpc31-Tyr4) or UAG (rpc31Tyr230) triplet. EMBL/GenBank accession number X51498.

Chromosome mapping. Since our data leave no doubt that $R P C 31$ encodes the C31 subunit of RNA polymerase C, we conserved the gene symbol $R P C 31$ initially proposed (39). This gene was assigned to chromosome XIV by the method of Falco and Botstein (10). Plasmid pJC31 (URA3 RPC31) was integrated into RPC31 in the MATa strain K382-23A by targeted integrative crossover (31). The resulting $R P C 31:$ : pJC31 (ura3 RPC31) transformant (strain KJ382-23A; Table 1) was crossed to three MATa ura3 ${ }^{-}$strains, K396-22B, K381-9D, and K393-35C, which together harbor at least one auxotrophy marker on each chromosome (21). The spontaneous ura3 segregants (formed at a frequency of about $2 \%$ ) were prototrophic for all but one (met4) of the heterozygous markers, thereby indicating that $R P C 31$, like $M E T 4$, maps on chromosome XIV. A more precise mapping was obtained by crossing the MATa RPC31::Y1pC327 (ura3 RPC31) strain DM32-1 or DM34-7d to the ura3 mutant strains DM33-1a

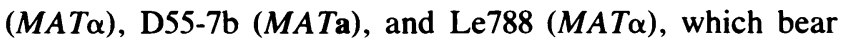
various genetic markers (pha2, petx, met2, and met4) on chromosome XIV (Table 1) (29). Tetrad analysis allowed us to localize $R P C 31$ on the left arm of the chromosome, between MET4 (23 parental ditypes, 22 tetratypes, and 3 recombinant ditypes) and PETX (8 parental ditypes and 5 tetratypes). The $M A K 26$ gene also maps in that region (49), but its precise position relative to that of $R P C 3 I$ has not been determined.

Amino acid sequence analysis. The calculated isoelectric point of the C31 subunit (assuming no polar modification of amino acids) is 4.5 . This is largely due to a highly acidic C-terminal domain, which mainly consists of Asp and Glu downstream from position 202 (Fig. 3). We compared the amino acid sequence of $\mathrm{C} 31$ to the most recent versions in the SWISSPRO, GENPRO, and NBRF data banks with the FASTA alignment program of Pearson and Lipman (34). The best alignment was obtained with the $\delta$ subunit (24) of the Bacillus subtilis RNA polymerase (initial score of 109 against an average score of $23.9 \pm 6.46$ ). Animal HMG1 proteins were also found among the 20 best alignments, with a rather modest initial score of 73 . All of the 20 best alignments corresponded to proteins with extensive stretches of acidic amino acids (Asp and Glu) and were limited to these stretches. Data bank searches done with the first 201 amino acids of RPC3I (without the acidic domain) as a probe yielded no appreciable homology to any known protein. We also noted that animal HMG1 proteins were strongly invariant except in their acidic tail, which is almost exclusively formed of Asp residues in the trout protein (35) but contains numerous Glu residues in the mammalian ones (32). No cross-antigenicity was detected between the C31 protein and animal HMG1 proteins by Western blot (immunoblot) analysis (data not shown). Finally, the cruciform DNA-binding activity typical of animal HMG1 proteins (3) was not detected on an in vitro translated $\mathrm{C} 31$ protein produced from plasmid pBSC31-5, with in vitro translated rat HMG1 as a positive control (data not shown).

Redundancy of the $3^{\prime}$-terminal part of RPC31 on the yeast genome. Southern hybridization of an RPC31 probe corresponding to the first 189 amino acids (without the acidic tail) yielded a single signal against the $S$. cerevisiae genome digested with various restriction enzymes (Fig. 4B), establishing that $R P C 31$ is a single-copy gene. There was no cross-hybridization against the $S$. pombe genome under low-stringency conditions (Fig. 4C), indicating that RPC 31 is at best poorly conserved between these two species, unlike the RPC160 gene encoding the largest subunit of RNA polymerase $C$ (data not shown). In contrast, a probe corresponding to the acidic tail of $R P C 31$ yielded numerous cross-hybridization signals against the $S$. cerevisiae and $S$. pombe genomes (Fig. 4D), indicating that both yeasts probably harbor numerous genes encoding proteins with highly acidic domains rich in Asp and Glu residues. In retrospect, this observation explains the fortuitous cloning of $R P C 31$ $(A C P 2)$ by Haggren and Kolodrubetz (15), who used a probe encoding a stretch of Asp and Glu residues to screen a yeast genomic library.

Mutagenesis of RPC31. The heterozygous diploid $r p c 31 \Delta:$ : LEU2/RPC31 was constructed by one-step disruption (42) of strain YNN281 $\times$ YNN282. The disrupted $r p c 31$ allele (detected by its $\mathrm{Leu}^{+}$phenotype) invariably cosegregated with a lethal phenotype (at most one or two cell divisions upon spore germination on the complete medium YPD at 16 and $30^{\circ} \mathrm{C}$ ) in tetrad analysis. Viable haploid segregants of the $r p c 31 \Delta:: L E U 2$ allele were recovered by transforming the heterozygous diploid with a centromeric plasmid bearing the 1.8-kb SpeI-EcoRI (pC50) or the 2.5-kb EcoRI-EcoRI (pC329 or pC326) fragment encompassing the RPC31 gene (Fig. 1B and C). These segregants could not lose the complementing plasmid, showing that the lethal phenotype reflects an inability to undergo vegetative growth and division and not just defective spore germination.

The rpc31 $1:: L E U 2$ allele deletes about 200 bp upstream from the $R P C 3 I$ coding region and may prevent the transcription of an essential gene adjacent to $R P C 31$, with $R P C 31$ itself encoding a nonessential product. To eliminate this possibility, we inactivated $R P C 31$ in gene disruption experiments with the $r p c 31:: \operatorname{Tn} 10-13$ and $r p c 31:: H I S 3 a$ insertions (Fig. 1C), which again yielded a recessive lethal phenotype cosegregating with the mutated allele. Further- 


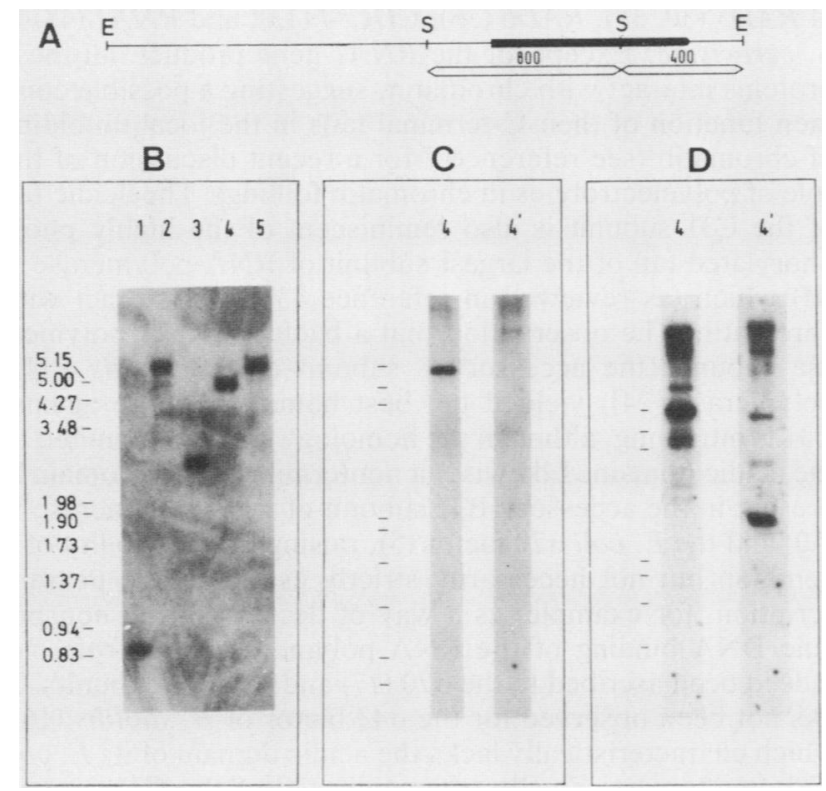

FIG. 4. Southern blot analysis of $S$. cerevisiae YNN281 and $S$. pombe $\mathrm{h}^{-} 972$ DNAs against $R P C 31$. (A) Simplified restriction map of the RPC31 region (2.5-kb EcoRI fragment; see Fig. 1B for restriction site symbols). The 800 -bp SalI fragment contains the first 189 codons of RPC31 (without the acidic tail). The 400-bp SallEcoRI fragment includes the acidic tail (see Fig. 3 for the position corresponding to the SalI restriction site in the amino acid sequence). (B) Stringent hybridization of the $S$. cerevisiae genome digested with different restriction enzymes against the 800-bp Sall probe. Lane numbers refer to the restriction enzymes used: 1, Sall; 2, NcoI; 3, EcoRI; 4, BamHI; 5, HpaI. (C) Hybridization of S. cerevisiae (lane 4) and $S$. pombe (lane $4^{\prime}$ ) DNAs (after BamHI digestion) against the 800-bp Sall probe under low-stringency conditions. Filters were washed twice in $4 \times$ SSC $(1 \times$ SSC is $0.15 \mathrm{M}$ $\mathrm{NaCl}$ plus $0.015 \mathrm{M}$ sodium citrate) for $10 \mathrm{~min}$ at room temperature and then for $1 \mathrm{~h}$ at $55^{\circ} \mathrm{C}$. The weak bands observed in the $S$. pombe DNA were due to a background signal corresponding to the rDNA restriction fragments. (D) Hybridization of the $S$. cerevisiae (lane 4) and $S$. pombe (lane $4^{\prime}$ ) DNAs (after BamHI digestion) against the 400-bp Sall-EcoRI probe containing the acidic tail of RPC3I under low-stringency conditions. Numbers at left are in kilobases.

more, we inactivated $R P C 31$ on plasmid pC329 by two additional insertions ( $r p c 31:: \operatorname{Tn} 10-33$ and $r p c 31:: \operatorname{Tn} 10-38)$ (Fig. 1C) and by six nonsense mutations (Fig. 3) scattered throughout $R P C 31$. The corresponding mutations (including an rpc31-Tyr4 (UAA) nonsense allele at the fourth codon of $R P C 31$ ) all failed to complement $r p c 31 \Delta:: L E U 2$, although they were themselves recessive, as established by a plasmid shuffle assay with plasmid pC50 (RPC3I) as the complementing plasmid. This result established that the lethal phenotype of the rpc31 $:: L E U 2$ allele is entirely due to its defective $R P C 31$ gene product. Finally, we noted that the C-terminal rpc31-TYR230 (UAG) allele (which deletes about half of the acidic C-terminal domain) also had a lethal phenotype, whereas the last six amino acids of the acidic tail were not essential, as they could be replaced by six unrelated amino acids by $r p c 31-H I S 3 b$ gene disruption without affecting the growth rate.

Defective tRNA synthesis in a leaky mutant. Two centromeric plasmids (pC329 and pC50) bearing RPC31 were mutagenized with hydroxylamine, and $R P C 31$ function was tested by genetic complementation of the $r p c 31 \Delta:: L E U 2$ allele in a plasmid shuffle test. We found no conditional (cold- or heat-sensitive) mutant under conditions that yield-

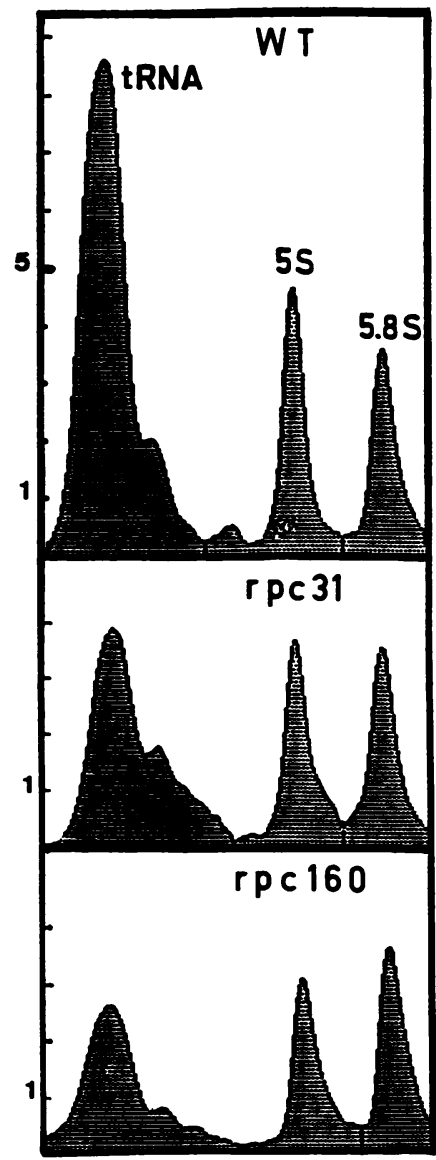

FIG. 5. Synthesis of small RNAs in a defective rpc31 mutant. The incorporation of ${ }^{3} \mathrm{H}$-labeled uracil into small RNAs was measured after $15 \mathrm{~min}$ of in vivo labeling at $30^{\circ} \mathrm{C}$ in strains YNN281 (wild type [WT]), D27-7c (rpc160-41), and DM824 (rpc31-Tyr4 SUP11-0) RNAs $(20 \mu \mathrm{g})$ were separated on a $6 \%$ polyacrylamide gel and autoradiographed. Uracil incorporation was quantified by densitometry of the autoradiogram after 3 to 10 days of exposure. The proportionality of the signals as a function of the exposure time was checked to avoid artifacts due to signal saturation. The heights of the peaks are given in arbitrary units and are normalized to the $5.8 \mathrm{~S}$ rRNA signal.

ed 225 fully defective mutants and thus probably corresponded to saturation mutagenesis. These data strongly suggest that temperature-sensitive alleles of $R P C 31$ are genuinely rare, at least after hydroxylamine mutagenesis. A leaky mutant strain was constructed by cloning the rpc31Tyr4 (UAA) insert on the centromeric plasmid pSE359, which bears the tyrosine-inserting suppressor SUP11-o. The resulting plasmid (pSE359-Tyr4) generated a wild-type protein with a reduced intracellular concentration due to the low efficiency of nonsense suppression. The resulting $r p c 31 \Delta$ :: LEU2 (pSE359-Tyr4) mutant (strain DM824) had a growth rate of $6 \mathrm{~h}$ on minimal medium supplemented with tryptophan and uracil at $30^{\circ} \mathrm{C}$, as compared with $2.5 \mathrm{~h}$ for our wild-type strain, YNN281, and with $3.0 \mathrm{~h}$ for the $\operatorname{rpc} 31 \Delta$ :: LEU2 (pSE359-RPC31 ${ }^{+}$) strain DM824.

We measured the incorporation of ${ }^{3} \mathrm{H}$-labeled uracil into small RNAs in strain DM824 after a short pulse of $15 \mathrm{~min}$ (Fig. 5; similar results were obtained after a 30-min pulse [data not shown]). The rpc160-41 mutant strain D27-7c (a bona fide RNA polymerase $\mathrm{C}$ mutant with a growth rate of $7 \mathrm{~h}$ at $\left.30^{\circ} \mathrm{C}[14]\right)$ was used as a control. Since tRNAs and 
rRNAs are comparatively stable molecules, this procedure essentially measures the rate of tRNA synthesis in vivo. To eliminate possible secondary effects of the reduced mutant growth rate per se on RNA polymerase $C$, we also reduced the growth of the wild-type control strain, YNN281, with glucosamine to compete for glucose uptake (27). The rpc3l and $r p c 160$ mutants both had reduced rates of tRNA synthesis (relative to 5.8S rRNA synthesis [Fig. 5]) that were correlated with their reduced growth rates. This result supports the hypothesis that the $C 31$ polypeptide is a functional subunit of RNA polymerase $C$. In keeping with earlier data on rpc160-4l (14), there was at best a minor effect on the rate of synthesis of $5 \mathrm{~S}$ rRNA.

\section{DISCUSSION}

We cloned a 13.5-kb fragment of the yeast genome that includes the single-copy gene $R P C 31$. DNA and protein sequence data demonstrated that it codes for the $C 31$ subunit of RNA polymerase C. Null mutations were constructed, and their lethality established that the integrity of this protein is essential for cellular growth and/or division. Furthermore, the reduced rate of tRNA synthesis of a partially defective mutant supported the hypothesis that the C31 protein functionally belongs to RNA polymerase $\mathrm{C}$. Unlike the genes of the large RNA polymerase subunits (51), RPC31 is not strongly conserved between the $S$. cerevisiae and $S$. pombe genomes. The gene was precisely located on the left arm of chromosome XIV, very close to an 8-bp NotI site. The only gene identified so far in that region (MAK26 [49]) controls the maintenance of the yeast RNA killer virus.

RPC31 codes for a 251-amino-acid protein with a predicted $M_{\mathrm{r}}$ of 27,707 and an isoelectric point of 4.5 . This acidic character is largely due to a $\mathrm{C}$-terminal domain which is characterized by stretches of Asp and Glu residues from positions 202 to 248 , which may be partly organized in a helix, and which is likely to protrude from the C31 protein because of its hydrophilic character and C-terminal position. Using an oligonucleotide probe rich in Asp and Glu codons, Haggren and Kolodrubetz (15) isolated the same gene (termed $A C P 2$, for acidic protein 2) which they interpreted as coding for an HMG1 protein. HMG1 proteins are functionally ill-defined components of chromatin which specifically bind cruciform DNA (3), a property that is not shared by the C31 protein. Furthermore, animal HMG1 proteins and the $\mathrm{C} 31$ subunit are antigenically unrelated, and there is no sequence homology outside a C-terminal run of Asp and Glu residues which creates a highly acidic domain also present in a number of other proteins (see below). This domain is itself not particularly invariant among animal HMG1 proteins. Taken together, these data strongly argue against the idea that $\mathrm{C} 31$ is an HMG1 protein. Interestingly, two $S$. cerevisiae genes (NHP6A and $N H P 6 B$ [22]) which code for polypeptides of about $\mathbf{1 0}$ kilodaltons were recently shown to have notable homology to the middle region of the animal HMG1 protein amino acid sequence. These polypeptides have no acidic tail and are in fact highly basic. The structure of yeast HMG1 proteins (and their functional relationship to their animal counterparts) remains, therefore, an open question.

The highly acidic C-terminal tail of the $\mathrm{C} 31$ protein is functionally or structurally essential, since the elimination of half of this domain by a nonsense mutation inactivated the protein in vivo. A C-terminal acidic domain is present on a variety of proteins, such as the spermine-binding protein (6), the murine homeodomain protein (20), and the gene products of $R A D 3(30,37), R A D 6(36), C D C 34(13)$, and $R N A I$ (48) in $S$. cerevisiae. Except for the RNAl gene product, all these proteins interact with chromatin, suggesting a possible common function of their C-terminal tails in the local unfolding of chromatin (see reference 7 for a recent discussion of the role of polyelectrolytes in chromatin folding). The acidic tail of the C31 subunit is also reminiscent of the highly phosphorylated tail of the largest subunit of RNA polymerase B (II) which (as reviewed in reference 43) may interact with chromatin. The observation that a bacterial RNA polymerase subunit (the accessory $\delta$ subunit of $B$. subtilis RNA polymerase [24]) yielded the best homology score against C31 is intriguing, although the homology was again limited to the acidic domain. Likewise, a nonterminal acidic domain is present in the accessory B32 subunit of RNA polymerase B (50) and the $E$. coli $\sigma 70$ factor (5), raising the possibility of a common but not necessarily strictly essential role in transcription, for example, as a way of decreasing the nonspecific DNA binding of the RNA polymerase. This role has indeed been ascribed to the $\sigma 70$ (17) and $\delta 21$ (47) subunits. It has not been observed for the $\sigma 43$ factor of $B$. subtilis (16), which characteristically lacks the acidic domain of its $E$. coli $\sigma 70$ counterpart. Finally, the acidic tail of the C31 protein may directly interact with a basic component of the RNA polymerase $\mathrm{C}$ transcription machinery, such as the TFIIIA transcription factor (12).

\section{ACKNOWLEDGMENTS}

We thank Pierre Fromageot for helpful support, Françoise Bouet for assistance in the oligopeptide sequencing, Jean-Marie Buhler and co-workers for preparing oligonucleotides, Dominique Lalo for contributing to the TnIO-LUK transposition experiment, Christian Marck for advice on sequence analysis, Robert Swanson for correcting our manuscript, and Michel Werner for particularly generous readiness to discuss our data. François Hilger, François Lacroute, and Carl Mann kindly provided unpublished strains and plasmids.

C.M. was supported by a Fellowship of the French Ministère de la Recherche Scientifique.

\section{LITERATURE CITED}

1. Allison, L. A., M. Moyle, M. Shales, and C. J. Ingles. 1985. Extensive homology among the largest subunit of eukaryotic and prokaryotic RNA polymerases. Cell 42:599-610.

2. Baldari, C., and G. Cesareni. 1985. Plasmids pEMBLY: new single stranded shuttle vectors for the recovery and analysis of yeast DNA sequences. Gene 35:27-32.

3. Bianchi, M. E., M. Beltrame, and G. Paonessa. 1989. Specific recognition of cruciform DNA by nuclear protein HMG1. Science 243:1056-1059.

4. Boeke, J. D., F. Lacroute, and G. R. Fink. 1984. A positive selection for mutants lacking orotidine-5'-phosphate decarboxylase activity in yeast: 5-fluoro-orotic acid resistance. Mol. Gen. Genet. 197:345-346.

5. Burton, Z., R. R. Burgess, J. Lin, D. Moore, S. Holder, and C. A. Gross. 1981. The nucleotide sequence of the cloned rpoD gene for the RNA polymerase sigma subunit from $E$. coli K12. Nucleic Acids Res. 9:2889-2903.

6. Chang, C., A. G. Saltzman, R. A. Hiipakka, I.-Y. Huand, and S. Liao. 1987. Prostatic-spermine binding protein. J. Biol. Chem. 262:2828-2831.

7. Clark, D. J., and T. Kimura. 1990. Electrostatic mechanism of chromatin folding. J. Mol. Biol. 211:883-896.

8. Davis, R. W., D. Botstein, and J. R. Roth. 1980. Advanced bacterial genetics, p. 254. Cold Spring Harbor Laboratory, Cold Spring Harbor, N.Y.

9. Elledge, S. J., and R. W. Davis. 1988. A family of versatile centromeric vectors designed for use in the sectoring-shuffle mutagenesis assay in Saccharomyces cerevisiae. Gene 70:303312. 
10. Falco, S. C., and D. Botstein. 1983. A rapid chromosome mapping method for cloned fragments of yeast DNA. Genetics 105:857-872.

11. Geiduschek, E. P., and G. P. Tocchini-Valentini. 1988. Transcription by RNA polymerase III. Annu. Rev. Biochem. 57: 873-914.

12. Ginsberg, A. M., B. O. King, and R. G. Roeder. 1984. Xenopus 5S gene transcription factor, TFIIIA: characterization of a cDNA clone and measurement of RNA levels throughout development. Cell 39:479-489.

13. Goebl, M. G., J. Yochem, S. Jentsch, J. P. McGrath, A. Varshavsky, and B. Byers. 1988. The yeast cell cycle gene CDC34 encodes a ubiquitin-conjugating enzyme. Science 241: 1331-1335.

14. Gudenus, R., S. Mariotte, A. Moenne, A. Ruet, S. Mémet, J.-M. Buhler, A. Sentenac, and P. Thuriaux. 1988. Conditional mutants of RPC160, the gene encoding the largest subunit of RNA polymerase C in Saccharomyces cerevisiae. Genetics 119:517526.

15. Haggren, W., and D. Kolodrubetz. 1988. The Saccharomyces cerevisiae ACP2 gene encodes an essential HMG1-like protein. Mol. Cell. Biol. 8:1282-1289.

16. Helmann, J. D., and M. J. Chamberlin. 1988. Structure and function of bacterial sigma factors. Annu. Rev. Biochem. 57: 839-887.

17. Hinkle, D. C., and M. C. Chamberlin. 1972. Studies of the binding of Escherichia coli RNA polymerase to DNA. J. Mol. Biol. 70:187-195.

18. Huet, J., M. Riva, A. Sentenac, and P. Fromageot. 1985. Yeast RNA polymerase $\mathrm{C}$ and its subunits: specific antibodies as structural and functional probes. J. Biol. Chem. 260:15304 15310 .

19. Huisman, O., W. Raymond, K. U. Froehlich, P. Errada, N. Kleckner, D. Botstein, and A. Hoyt. 1987. A Tnl0-lacZ-kandR$U R A 3$ gene fusion transposon for insertion mutagenesis and fusion analysis of yeast and bacterial genes. Genetics 116:191199.

20. Kessel, M., F. Schultze, M. Fibi, and P. Gruss. 1987. Primary structure and nuclear localization of a murine homeodomain protein. Proc. Natl. Acad. Sci. USA 84:5306-5310.

21. Klapholz, S., and R. Easton-Esposito. 1980. A new mapping method employing a meiotic rec mutant of yeast. Genetics 96:589-611.

22. Kolodrubetz, D., and A. Burgum. 1990. Duplicated NHP6 genes of Saccharomyces cerevisiae encode proteins homologous to bovine high mobility group protein 1 . J. Biol. Chem. 265:3234 3239.

23. Kolodziej, P., and R. A. Young. 1989. RNA polymerase II subunit RPB3 is an essential component of the mRNA transcription apparatus. Mol. Cell. Biol. 9:5387-5394.

24. Lampe, M., C. Binnie, R. Schmidt, and R. Losick. 1988. Cloned gene encoding the delta subunit of Bacillus subtilis. Gene 67:13-19.

25. Mann, C., J. M. Buhler, I. Treich, and A. Sentenac. 1987. $R P C 40$, a unique gene for a subunit shared between yeast RNA polymerase A and C. Cell 48:627-637.

26. Marck, C. 1988. "DNA Strider": a C program for the fast analysis of DNA and protein sequences on the Apple Macintosh family of computers. Nucleic Acids Res. 16:1829-1836.

27. McGoldrick, E. M., and A. E. Wheals. 1989. Controlling the growth rate of Saccharomyces cerevisiae cells using the glucose analogue D-glucosamine. J. Gen. Microbiol. 135:2407-2411.

28. Mémet, S., M. Gouy, C. Marck, A. Sentenac, and J.-M. Buhler. 1988. RPA190, the gene for the largest subunit of yeast RNA polymerase A. J. Biol. Chem. 263:2830-2839.

29. Mortimer, R. K., D. Schild, C. R. Contopoulou, and J. A. Kans. 1989. Genetic map of Saccharomyces cerevisiae. Yeast 5:321403.

30. Naumovski, L., G. Chu, P. Berg, and E. C. Friedberg. 1985. RAD3 gene of Saccharomyces cerevisiae: nucleotide sequence of wild-type and mutant alleles, transcript mapping, and aspects of gene regulation. Mol. Cell. Biol. 5:17-26.
31. Orr-Weaver, T. L., J. W. Szostak, and R. J. Rothstein. 1981. Yeast transformation: a model for the study of recombination. Proc. Natl. Acad. Sci. USA 78:6354-6358.

32. Paonessa, G., R. Frank, and R. Cortese. 1987. Nucleotide sequence of rat liver HMG-1 cDNA. Nucleic Acids Res. 15: 9077-9087.

33. Parent, S. A., C. M. Fenimore, and K. A. Bostian. 1985. Vector systems for the expression, analysis and cloning of cDNA sequences in $S$. cerevisiae. Yeast 2:83-138.

34. Pearson, W. R., and D. J. Lipman. 1988. Improved tools for biological sequence comparison. Proc. Natl. Acad. Sci. USA 85:2444-2448.

35. Pentecost, B. T., J. M. Wright, and G. H. Dixon. 1985. Isolation and sequence of cDNA clones coding for members of the family of high mobility group of protein (HMG-T) in trout and analysis of HMG T mRNA's in trout tissues. Nucleic Acids Res. 13:4871-4887.

36. Reynolds, P., D. R. Higgins, L. Prakash, and S. Prakash. 1985. RAD6 gene of Saccharomyces cerevisiae encodes a protein containing a tract of 13 consecutive aspartates. Nucleic Acids Res. 13:2357-2372.

37. Reynolds, P., S. Weber, and L. Prakash. 1985. The nucleotide sequence of $R A D 3$ gene of Saccharomyces cerevisiae: a potential adenine nucleotide binding amino-acid sequence and a nonessential acidic carboxyl terminal region. Proc. Natl. Acad. Sci. USA 82:168-172.

38. Riva, M., J.-M. Buhler, A. Sentenac, P. Fromageot, and D. C. Hawthorne. 1982. Natural variation in yeast RNA polymerase A. Formation of a mosaic RNA polymerase $A$ in a meiotic segregant from an interspecific hybrid. J. Biol. Chem. 257:4570 4577.

39. Riva, M., S. Mémet, J.-Y. Micouin, J. Huet, I. Treich, J. Dassa, R. Young, J.-M. Buhler, A. Sentenac, and P. Fromageot. 1986. Isolation of structural genes for yeast RNA polymerases by immunological screening. Proc. Natl. Acad. Sci. USA 83:1554 1558.

40. Rose, M. D., and G. R. Fink. 1987. KAR1, a gene required for function of both intranuclear and extranuclear microtubules. Cell 48:1047-1060.

41. Rose, M. D., P. Novick, J. H. Thomas, D. Botstein, and G. R. Fink. 1987. A Saccharomyces cerevisiae genomic plasmid bank based on a centromere-containing shuttle vector. Gene 60:237243.

42. Rothstein, L. R. 1983. One step gene disruption in yeast. Methods Enzymol. 101:202-210.

43. Sawadogo, M., and A. Sentenac. 1990 . RNA polymerase B (II) and general transcription factors. Annu. Rev. Biochem. 59:711754.

44. Sentenac, A. 1985. Eukaryotic RNA polymerases. Crit. Rev. Biochem. 18:31-91.

45. Sherman, F., G. R. Fink, and J. B. Hicks. 1986. Laboratory course manual for methods in yeast genetics. Cold Spring Harbor Laboratory, Cold Spring Harbor, N.Y.

46. Sweetser, D., M. Nonet, and R. A. Young. 1987. Prokaryotic and eukaryotic RNA polymerases have homologous core subunits. Proc. Natl. Acad. Sci. USA 84:1192-1196.

47. Tjian, R., R. Losick, J. Pero, and A. Hinnebush. 1977. Purification and comparative properties of delta and sigma subunits of RNA polymerase from Bacillus subtilis. Eur. J. Biochem. 74:149-154.

48. Traglia, H. M., N. S. Atkinson, and A. Hopper. 1989. Structural and functional analyses of Saccharomyces cerevisiae wild-type and mutant RNAl genes. Mol. Cell. Biol. 9:2989-2999.

49. Wickner, R. B. 1979. Mapping chromosomal genes of Saccharomyces cerevisiae using an improved genetic mapping method. Genetics 92:803-821.

50. Woychik, N. A., and R. A. Young. 1989. RNA polymerase II subunit RPB4 is essential for high- and low-temperature yeast cell growth. Mol. Cell. Biol. 9:2854-2859.

51. Yamagishi, M., and M. Nomura. 1988. Cloning and sequence determination of the largest subunit of the fission yeast Schizosaccharomyces pombe RNA polymerase I. Gene 74:503-515. 